\title{
Multipoint Optimal Minimum Entropy Deconvolution Adjusted for Automatic Fault Diagnosis of Hoist Bearing
}

\author{
Tengyu Li, ${ }^{1,2}$ Ziming Kou $\mathbb{D}^{1,2}{ }^{1,2}$ Juan Wu, ${ }^{1,2}$ Waled Yahya, ${ }^{1,2}$ and Francesco Villecco ${ }^{3}$ \\ ${ }^{1}$ College of Mechanical and Vehicle Engineering, Taiyuan University of Technology, Taiyuan 030024, China \\ ${ }^{2}$ Shanxi Province Engineering Technology Research Center for Mine Fluid Control, Taiyuan 030024, China \\ ${ }^{3}$ Department of Industrial Engineering, University of Salerno, Via Giovanni Paolo II, 132, 84084 Fisciano, SA, Italy
}

Correspondence should be addressed to Ziming Kou; zmkou@163.com

Received 1 November 2020; Revised 13 December 2020; Accepted 24 December 2020; Published 15 January 2021

Academic Editor: Liang Guo

Copyright (c) 2021 Tengyu Li et al. This is an open access article distributed under the Creative Commons Attribution License, which permits unrestricted use, distribution, and reproduction in any medium, provided the original work is properly cited.

\begin{abstract}
Multipoint optimal minimum entropy deconvolution adjusted (MOMEDA) is a powerful method that can extract the periodic characteristics of signal effectively, but this method needs to evaluate the fault cycle a priori, and moreover, the results obtained in a complex environment are easily affected by noise. These drawbacks reduce the application of MOMEDA in engineering practice greatly. In order to avoid such problems, in this paper, we propose an adaptive fault diagnosis method composed of two parts: fault information integration and extracted feature evaluation. In the first part, a Teager energy spectrum amplitude factor (T-SAF) is proposed to select the intrinsic mode function (IMF) components decomposed by ensemble empirical mode decomposition (EEMD), and a combined mode function (CMF) is proposed to further reduce the mode mixing. In the second part, the particle swarm optimization (PSO) taking fractal dimension as the objective function is employed to choose the filter length of MOMEDA, and then the feature frequency is extracted by MOMEDA from the reconstructed signal. A cyclic recognition method is proposed to appraise the extracted feature frequency, and the evaluation system based on threshold and weight coefficient removes the wrong feature frequency. Finally, the feasibility of the method is verified by simulation data, experimental signals, and on-site signals. The results show that the proposed method can effectively identify the bearing state.
\end{abstract}

\section{Introduction}

The floor-type multirope friction hoist system is an important equipment in coal mine production. Its critical rotating parts (main shaft and sheave) have the critical task of transmitting power and supporting the load. Once a bearing fault occurs, it is effortless to cause serious safety accidents. Therefore, the study of bearing automatic fault diagnosis is very important for a safe operation of the hoisting system $[1,2]$.

In engineering practice, the hoist bearing has several unique characteristics in comparison to common bearings, such as heavy load (tens or even hundreds of tons), large size (diameter of meters), and low rotating speed (40-60 rpm) [3]. The low-speed and heavy-load characteristics of bearing make the fault signal weak and easily disturbed by noise $[4,5]$. Moreover, the running state of hoist bearing is often unknown in practice. Although intelligent diagnosis methods have apparent effects based on learning and classification, they need to deal with complex algorithms and collect field fault data to get accurate diagnosis results [6-9]. However, for security reasons, it is extremely hard to obtain the different fault data of hoist bearing in actual working conditions. Because of the above problems, a simple method composed of fault information integration and extracted feature evaluation is proposed to obtain the fault features and realize an automatic diagnosis.

The vibration signal is a nonstationary signal with complex frequency components, where the characteristic frequency representing the state of bearing can be extracted through a variety of methods [10-14]. The ensemble empirical mode decomposition (EEMD) is widely used in rotating body fault diagnosis [15]. Although it is difficult for EEMD to directly obtain satisfactory results from the 
vibration signal with complex components, this method can decompose a large amount of useful information into a single intrinsic mode function (IMF), called optimal IMF, making it easy to get the characteristic frequency. Therefore, to select the optimal IMF accurately, many indexes have been proposed [16-19]. Yang et al. used the average mutual information to select the "true" IMFs and the minimum sample entropy to choose the effective IMFs [20]. Still, this method is computationally complex and selects the IMF with the least noise rather than the most fault information. $\mathrm{Wu}$ et al. took kurtosis as the index and three as the threshold to obtain the reconstructed signal by adding the eligible IMFs [21]. However, kurtosis is easily affected by noise and is unstable [22]. Yang et al. defined the spectral amplification factor calculated from Fourier spectrum and fault frequency band of the IMFs and raw signal to select the optimal IMF [23]. This index is simple to calculate and has little noise interference, but the fault information is mainly concentrated at the frequency doubling in the Fourier spectrum [24]. So, a lot of fault information may be lost by selecting the optimal IMF component through the fault frequency band. Also, it is impossible to select the range of fault frequency band a priori when the bearing state is unknown. Consequently, the Teager energy spectral amplitude factor (T-SAF) is built to choose the optimal IMFs corresponding to different fault types, and a combined mode function (CMF) is proposed to get the reconstructed signal and solve the problem of mode mixing.

Multipoint optimal minimum entropy deconvolution adjusted (MOMEDA) can extract cyclical features without iteration. It uses a target vector constructed according to the theoretical fault frequency to determine the locations and weightings of the periodic characteristics [25]. It is necessary to select the appropriate filter length and cycle search range. Yao et al. used a periodic modulation intensity to estimate the fault cycle and approximated the real fault cycle by multiple iterations and proposed a method to solve the filter through particle swarm optimization (PSO) algorithm [26, 27]. Wang et al. evaluated the signals obtained under different filter lengths through the kurtosis spectral entropy so as to determine the optimal filter length [28]. For the hoist bearing, the low rotation speed and large structure size make the theoretical fault frequencies often located in a small range. When an appropriate sampling frequency is selected, the cycles of different faults are located in a small range. Thus, we can determine the search range according to this range and use PSO to choose the filter length. When the effect of EEMD is good, the method can extract the correct frequency, but the efficiency of EEMD is greatly affected by the level of white Gaussian noise [29]. Therefore, a cyclic recognition method based on frequency ratio coefficient and weight is proposed to quantitatively evaluate the extracted feature frequency, so as to realize the automatic recognition of bearing status. In each iteration, the weight coefficient is continuously updated by comparing the frequency ratio coefficient with the preset threshold, thereby excluding the wrong frequency and determining the fault type.
The remainder of this paper is organized as follows. In Section 2, the basic theory and the proposed method are explained. Section 3 verifies the method by different bearing fault experimental signals. Section 4 applies the proposed method to analyze the field signals of sheave bearing. Section 5 gives the conclusion.

\section{Theory and Analysis}

2.1. EEMD Theory and Fault Diagnosis Process. EEMD can solve mode mixing and endpoint effect by superposing the white Gaussian noise. The EEMD method first needs to determine the parameters of the added white noise, that is, the level and amount of white noise. Then, EMD is carried out to decompose the new signal by adding white noise to the raw signal, and multigroup IMFs are obtained by repeating the process. Finally, the signals of averaging the same IMFs of all groups are used as final results. In general, the amplitude of white noise is 0.2 times the standard deviation of the original signal, but according to reference [30], the white noise level $c=0.2$ does not apply to all signals. The larger white noise level will cause the phenomenon of overdecomposition, and the smaller white noise will not change the extreme point position. So, an iterative increase method of the white noise level is used to raise the decomposition efficiency of EEMD. The specific process is shown in Figure 1. In each iteration, the white noise level is increased by adding the step size $(h=0.01)$ and EEMD is carried out. Then, the feature frequency is extracted by the CMF method and PSO-MOMEDA. Finally, automatic diagnosis is realized by the evaluation system.

\subsection{Combined Mode Function (CMF) Method. EEMD can} decompose vibration signal into multiple IMFs adaptively, and each IMF has only one fixed frequency band. But the main energy of the fault signal is concentrated at the frequency doubling in Fourier frequency spectrum. Therefore, the fault characteristics are to be distributed to different IMFs. By comparing the amplitude of different IMFs in the same fault frequency band, the optimal IMF corresponding to this fault type can be determined. The Teager energy operator (TEO) can enhance the amplitude of the signal to a greater extent [31]. Therefore, the TEO is adopted to further strengthen the impact signal and make the fault frequency more obvious. In general, the Teager energy spectrum amplitude factor (T-SAF) is constructed to evaluate IMFs, and the optimal IMFs can be determined based on maximum T-SAF. The mathematical expression of T-SAF is shown as follows:

$$
S=\frac{\sum_{f=f_{n}-\delta}^{f_{n}+\delta} A_{\mathrm{imf}}(f)}{\sum_{f=f_{n}-\delta}^{f_{n}+\delta} A_{x}(f)},
$$

where $A_{\text {imf }}(f)$ is the amplitude in the envelope spectrum of the IMF, $A_{x}(f)$ is the amplitude in the envelope spectrum of the original signal, $f_{n}$ represents the theoretical fault frequency, and $2 \delta$ is the frequency bandwidth. 


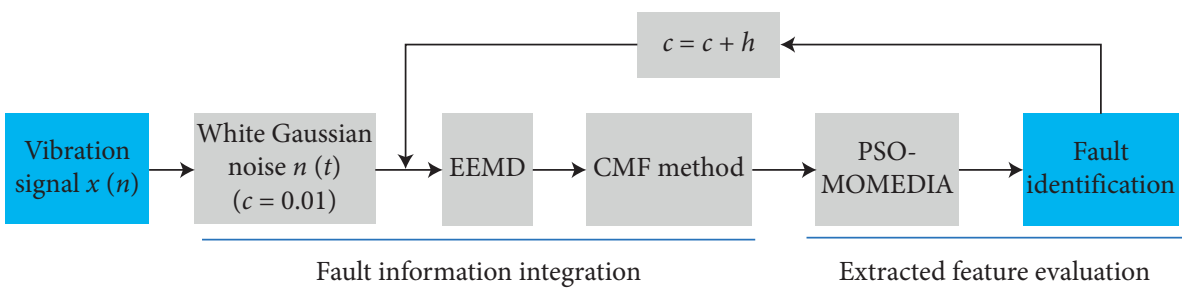

Figure 1: Fault identification process.

When the fault type is determined, the optimal IMF can be easily identified. Still, the running state of the hoist bearing in engineering practice is unknown, so the range of frequency band cannot be selected in advance. Considering the low-frequency characteristics of the bearing, a combined mode function method is proposed to reconstruct the signal including all fault characteristic frequencies, as shown in Figure 2. The optimal IMFs corresponding to inner ring fault, outer ring fault, rolling element fault, and the normal condition are added to form a reconstructed signal.

In order to show the availability of the CMF, the bearing fault model in reference [23] is adopted to establish the inner ring fault simulation signal. The structural parameters of hoist bearing in actual working conditions are given in Table 1. The bearing rotation frequency $f_{r}$ is $0.87 \mathrm{~Hz}$, and the fault frequency of the inner ring is $7.91 \mathrm{~Hz}$, which is approximately 9 times the rotation frequency $\left(9 f_{r}=7.83 \mathrm{~Hz}\right)$. The outer ring fault frequency $f_{o}$ is $6.01 \mathrm{~Hz}$, which is about 7 times the rotation frequency $\left(7 f_{r}=6.09 \mathrm{~Hz}\right)$. The rolling element fault frequency is $3.13 \mathrm{~Hz}$. The sampling frequency $f_{s}$, the sampling length $N$, and the bandwidth $2 \delta$ are set as $512 \mathrm{~Hz}, 4000$ samples, and $1 \mathrm{~Hz}$, respectively. The fault signal and its Fourier transform spectrum are shown in Figure 3(a). The rotation frequency, inner ring fault frequency, frequency doubling, and side frequencies are apparent, and the maximum amplitude appears at $70.91 \mathrm{~Hz}\left(9 f_{i}\right)$. The vibration signal formed by superposing noise to the fault signal is shown in Figure 3(b). The characteristic frequency is entirely submerged by noise, so it is hard to analyze the bearing state.

EEMD is used to decompose the simulation signal to obtain IMF components, and then the T-SAF values are calculated and normalized, as shown in Figure 4. The optimal IMFs corresponding to different bearing states are IMF3, IMF4, IMF4, and IMF7, as shown in Figure 5. In IMF3, the characteristic frequency of $8.19 \mathrm{~Hz}$ appears near the theoretical inner ring fault frequency of $f_{i}=7.91 \mathrm{~Hz}$, and there is also an interference frequency of $6.01 \mathrm{~Hz}$. In IMF4, the frequencies of 6.14 and $3.02 \mathrm{~Hz}$ are displayed clearly, and the inner ring fault frequency is completely submerged. In IMF7, the characteristic frequency of $0.77 \mathrm{~Hz}$ is close to the rotation frequency of $f_{r}=0.87 \mathrm{~Hz}$. The reconstructed signal formed by the CMF method is shown in Figure 6. The frequencies of $1.79,8.19$, and $11.52 \mathrm{~Hz}$ correspond to the theoretical characteristic frequencies of $2 f_{r}=1.74 \mathrm{~Hz}$, $f_{i}=7.91 \mathrm{~Hz}$, and $13 f_{r}=11.31 \mathrm{~Hz}$, respectively. Although the fault frequency is obvious in the reconstructed signal, the interference frequencies are not completely eliminated,

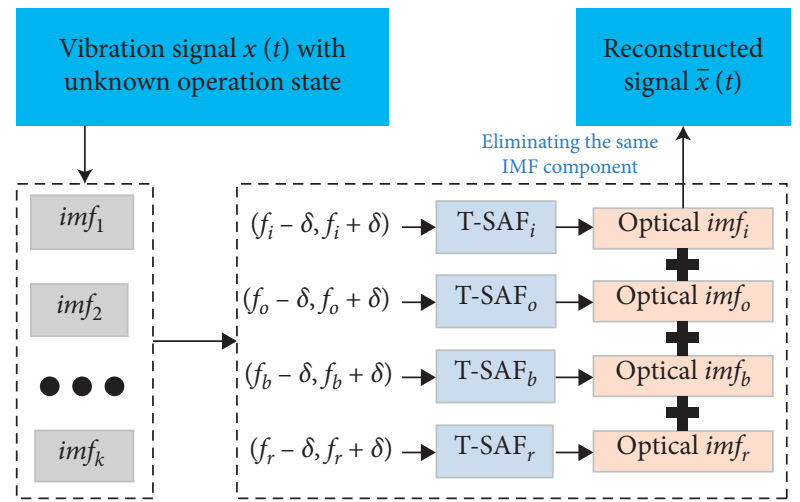

FIGURE 2: Signal reconstruction flow diagram.

which can easily cause misdiagnosis. Therefore, the MOMEDA method is adopted to denoise the reconstructed signal.

2.3. PSO-MOMEDA Algorithm. The basic theory of MOMEDA is in reference [25]. Its purpose is to find optimal filter without iteration and highlight the shock signals within a specific time scale. The effect of MOMEDA is related to the fault cycle and filter length greatly. In order to obtain the characteristic frequency more accurately, appropriate sampling frequency is defined to make the fault cycles of bearing with low-frequency characteristic in a smaller range, multipoint kurtosis is used as a metric for determining the period, and the PSO algorithm is used to select the filter length. The specific optimization process is as follows:

(1) Initialize input parameters, such as particle position (filter length), initial particle number, number of iterations, and so on.

(2) Based on the maximum multipoint kurtosis, the optimal fault cycle is determined and the noise reduction signal is obtained, and then the objective function is used to evaluate the noise reduction signal.

(3) Update the particle position and velocity and save the optimal position of each particle and the global optimal position, until the iteration termination condition is met.

(4) Use MOMEDA to reduce noise according to the optimized parameters and then extract the characteristic frequency. 
TABLE 1: The parameter of the hoist bearing SKF241/630 CAK/W33.

\begin{tabular}{lcccc}
\hline Rotating ring & Rotation speed & Ball diameter & Number of balls & Pitch diameter \\
\hline Inner ring & 52 & 85.73 & 16 & 628.65 \\
\hline
\end{tabular}
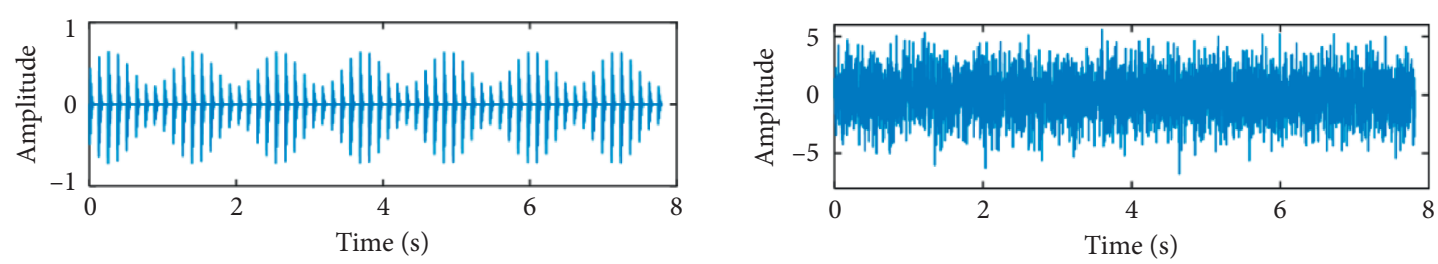

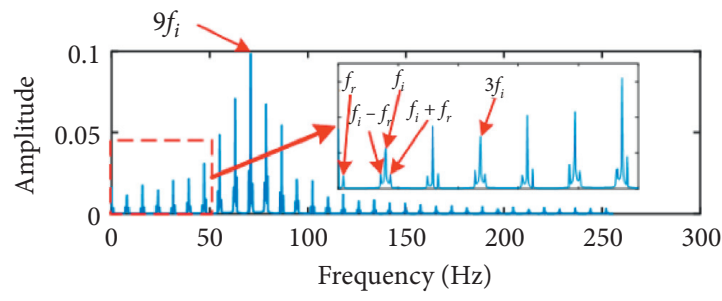

(a)

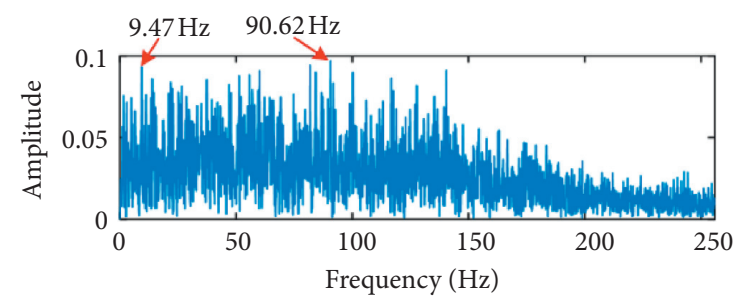

(b)

FIGURE 3: The inner ring fault simulation signal: (a) no noise added; (b) noise added.

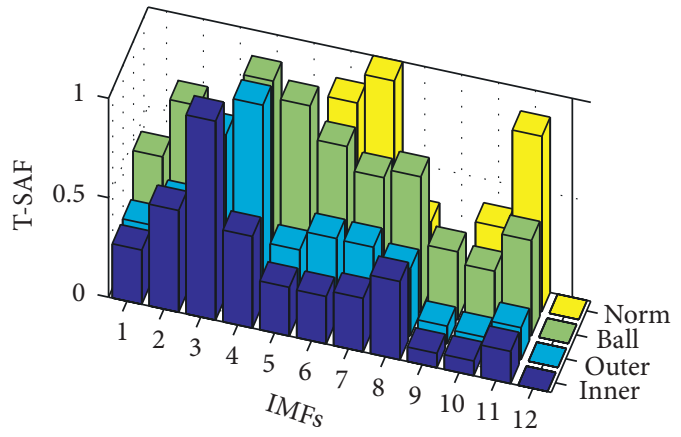

Figure 4: T-SAF values of the IMFs.

The fractal dimension describes the complexity and roughness of the curve, which is a useful index for analyzing bearing vibration signal [32]. We used fractal dimension as the objective function. In order to show the effectiveness of this index, it is compared with the kurtosis and permutation entropy. Taking the above simulation signal as an example, the theoretical fault cycles of the inner ring, outer ring, and rolling element are $65.74,85.18$, and 163.56 , respectively, so the search range is in the interval [50 170]. The comparison of indexes is shown in Figure 7. The frequency difference is the difference between the extracted characteristic frequency and the theoretical inner ring fault frequency. The characteristic frequencies corresponding to the maximum kurtosis, minimum permutation entropy, and minimum fractal dimension are $8.19,8.19$, and $8.09 \mathrm{~Hz}$, respectively, but only the fractal dimension has a similar trend with the frequency difference.

The PSO method is used to optimize the MOMEDA parameter. Set the initial particle swarm number to 20 , the number of iterations to 10 , and the filter length range in the interval [400 1000]. The calculated optimal filter length is 625, and the filtered signal is shown in Figure 8; the solid blue line representing the filtered signal almost corresponds to the red dashed line representing the fault signal, and the characteristic frequency is $7.89 \mathrm{~Hz}$, which is closer to the theoretical inner ring fault frequency than the frequencies shown in Figure 7.

2.4. Cyclic Recognition Method. According to the theory of the MOMEDA algorithm, the peak of the signal after noise reduction is usually a multiple of the fault period [28]. And improper white noise level will make MOMEDA extract the wrong characteristic frequency. The ability to manually identify the bearing fault type based on the extracted characteristic frequency is limited to a certain extent by the experience and knowledge of the field staff. Therefore, a cyclic recognition method is proposed according to threshold and weight. The specific process is shown in Figure 9.

According to the noise reduction principle of MOMEDA, the extracted characteristic frequency may be multiple of fault frequency [18]. Therefore, the frequency doubling coefficient between the characteristic frequency and fault frequency is first calculated to determine the fault type:

$$
C=\operatorname{round}\left(\frac{f_{m}}{f_{f}}\right)
$$

where round stands for rounding operation, $f_{m}$ is the characteristic frequency, and $f_{f}$ is the theoretical fault frequency. In order to realize automatic identification of fault types, the results need to be quantified. Based on the relative error calculation method, the frequency ratio is defined, and its formula is as follows: 

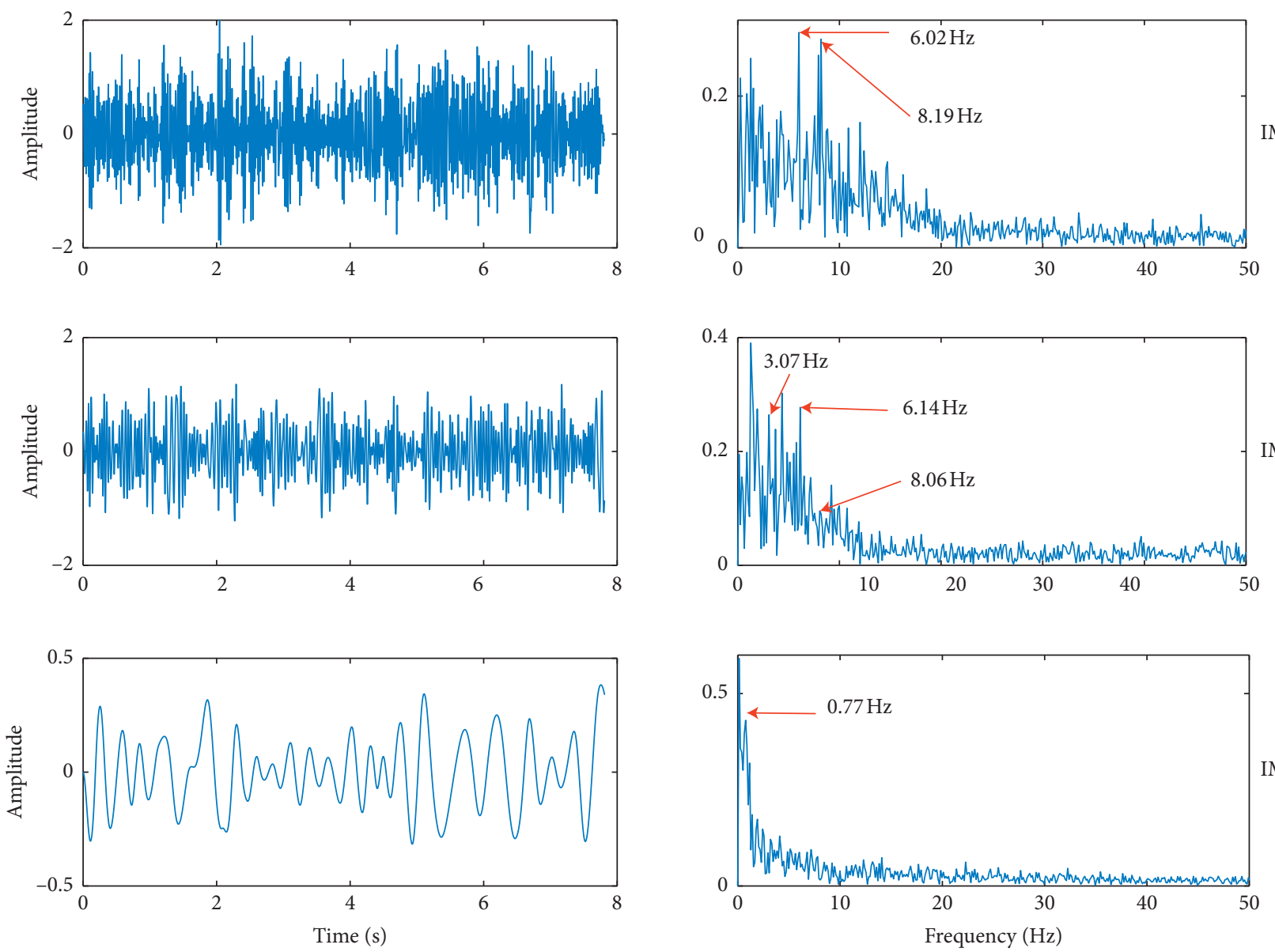

FIgURE 5: The optimal IMF selected according to the maximum T-SAF.
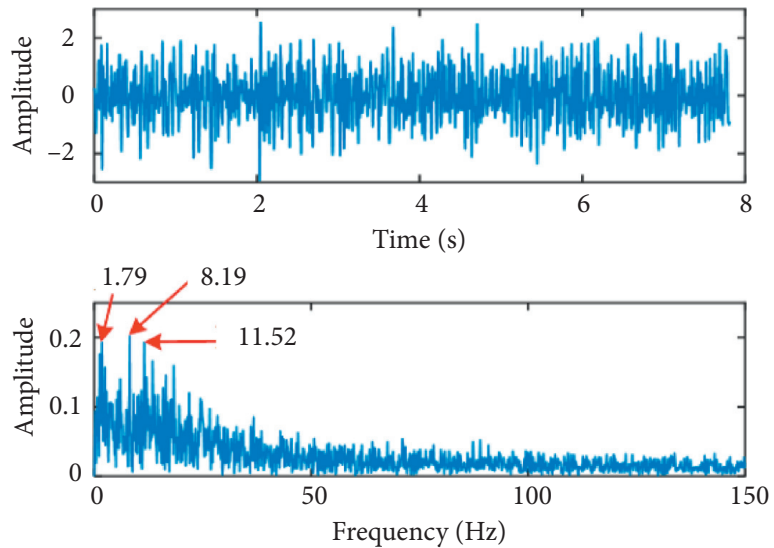

FIgURE 6: Time-domain waveform and envelope spectrum of the reconstructed signal.

$$
D=\frac{\operatorname{abs}\left(f_{m}-C \times f_{f}\right)}{f_{f}},
$$

where abs represents the absolute value. Finally, the frequency ratio corresponding to different fault types is compared with the preset threshold to realize the bearing

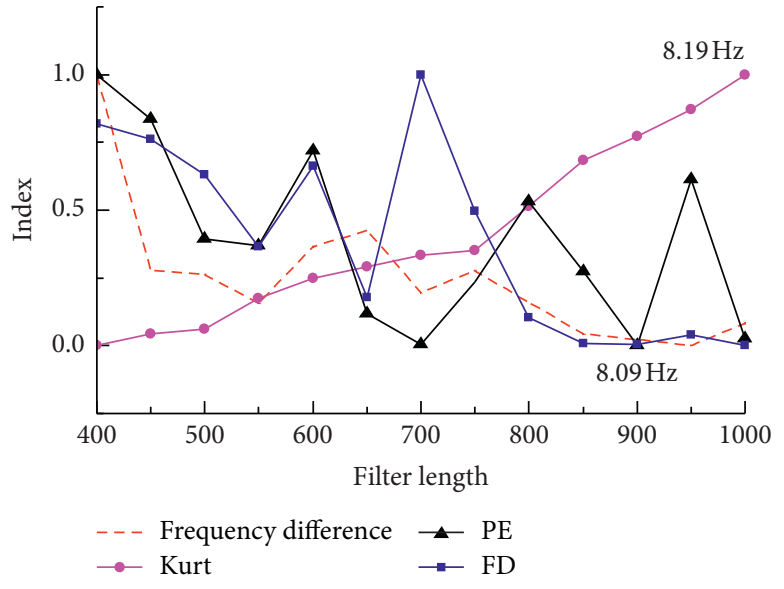

Figure 7: Comparison of different indexes.

state diagnosis. Considering that using the same threshold for different fault frequencies may make the diagnosis wrong, an adaptive threshold is proposed as the judgment standard. The threshold is linearly changed based on the inner ring fault frequency, and the specific calculation is shown in the following equation: 


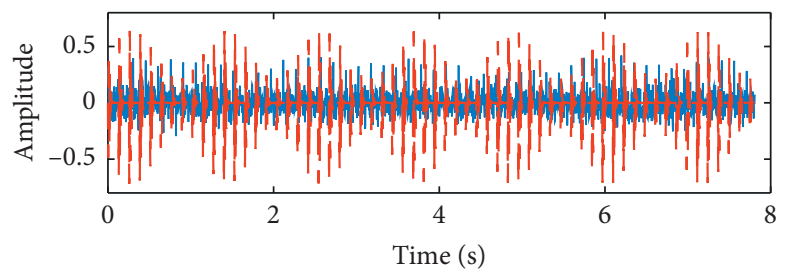

— Signal after MOMEDIA

- - - Fault signal

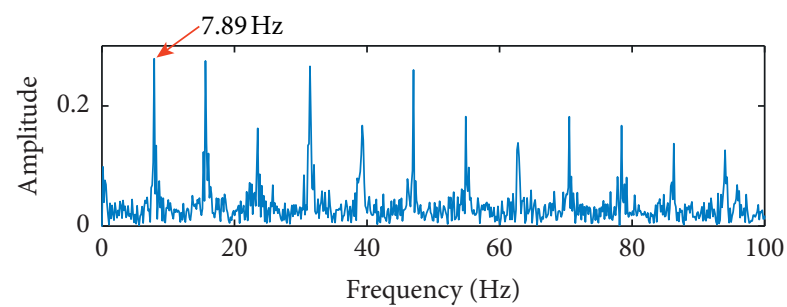

Figure 8: The filtered signal after MOMEDA.

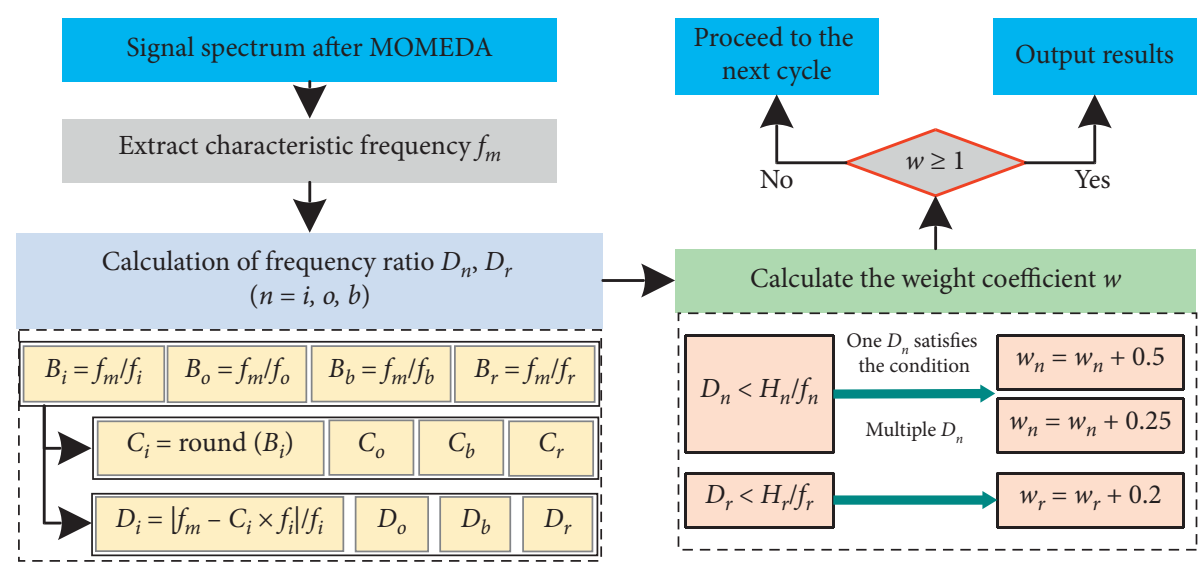

FIgUre 9: Automatic fault identification process.

$$
\left\{\begin{array}{l}
H_{1}=\frac{H \times f_{m}}{f_{i}}\left(H_{1} \leq H\right), \\
H_{1}=H \quad\left(H_{1}>H\right), \\
S=\frac{H_{1}}{f_{f}}
\end{array}\right.
$$

$H$ is an estimated value, which represents the maximum difference between the real fault frequency and the theoretical fault frequency; its value can refer to the frequency bandwidth, generally $\delta / 2 . H_{1}$ is the threshold after linear change, and its maximum value does not exceed $H$.

Due to the low-frequency characteristics and structural characteristics of the hoisting bearing, some fault frequencies have a certain multiple relationship with the rotation frequency. For example, $f_{i}=7.91 \mathrm{~Hz}$ and $9 f_{r}=7.83 \mathrm{~Hz}$. This makes the quantitative results meet the threshold requirements of multiple fault types at the same time, which brings greater difficulties to fault identification. Therefore, in order to avoid the bearing state recognition errors, the weight coefficient is established to evaluate each recognition result, as shown in the following equation:

$$
\left\{\begin{array}{cl}
w_{f}^{1}=w_{f}^{1}+0.5 & \left(D_{n}^{1}<S\right), \\
w_{f}^{m}=w_{f}^{m}+0.25 & \left(D_{n}^{m}<S\right), \\
w_{r}=w_{r}+0.2 & \left(D_{r}<S_{r}\right) .
\end{array}\right.
$$

$D_{n}{ }^{1}$ means that only one bearing state is identified; thus, the fault weight corresponding to the fault type in this cycle is set to $0.5 . D_{n}{ }^{m}$ multiple bearing states have been identified and accurate results have not been obtained; thus, the failure weight coefficients of all identified fault types are set to $0.25 . w_{r}$ is 0.2 when the frequency ratio is less than the normal state threshold. When the weight coefficient reaches 1 , the cycle stops, and the corresponding fault type is output.

The frequency obtained from the filtered signal in each cycle is shown in Figure 10(a); the abscissa is cycle number, the ordinate is the characteristic frequency, and the red dotted line indicates the theoretical fault frequency value corresponding to the fault type. The frequency ratios are 


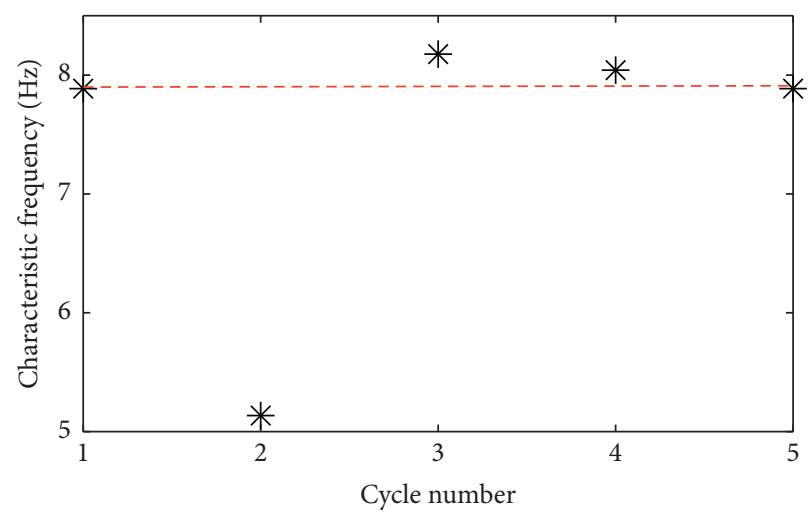

(a)

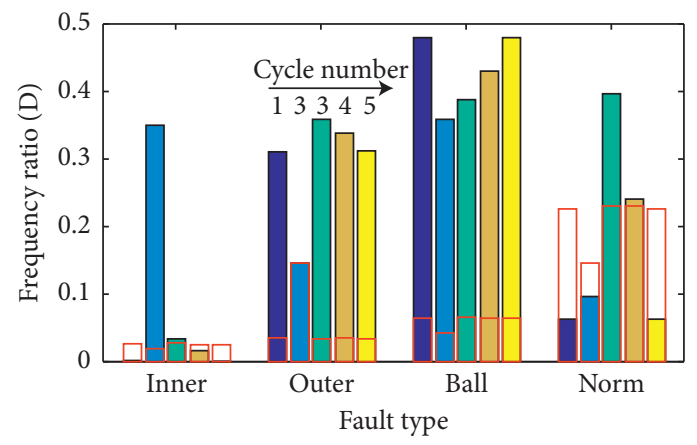

(b)

FIgURe 10: Automatic fault identification. (a) Characteristic frequency in each cycle. (b) The frequency ratio of fault types in each cycle.

shown in Figure 10(b); the abscissa is the fault type, the ordinate represents the frequency ratio, and the red line indicates the threshold for each fault type; $H=0.2 \mathrm{~Hz}$ is set here. The figure shows that the characteristic frequencies of 7.89, 8.04, and $7.89 \mathrm{~Hz}$ obtained in the first, fourth, and fifth cycles are less than the set thresholds. Among them, $7.89 \mathrm{~Hz}$ corresponds to both the inner ring fault and the normal state, and $8.04 \mathrm{~Hz}$ only corresponds to inner ring fault. According to equation (5), the weight coefficient of the inner ring is 1 after the fifth cycle, so it is determined as the inner ring fault. The simulation result illustrates the feasibility of the method, which is further verified by experiments.

\section{Experimental Verification}

3.1. Algorithm Flow. In order to realize the fault diagnosis of bearings with the unknown state in actual working conditions, a fault diagnosis method is proposed, as shown in Figure 11.

3.2. Experiment Rig and Conditions. The coal mine hoist has strict requirements on safety, so it is hard to implement onsite bearing failure tests. To verify the proposed method, the hoist simulation testing setup shown in Figure 12 was built. Figure 12(a) shows a schematic diagram of the hoist simulation testing setup, which mainly includes the steel structure support, wire rope, hoisting container, guide wheel, driving mechanisms, anti-running rope device, and hydraulic station. The $10 \mathrm{~m}$ high structure support is used to simulate the derrick, and the guide wheel is used to simulate the hoisting sheave. The driving mechanism provides driving force through a three-phase asynchronous motor and controls the running direction and speed of the steel wire rope through a matched frequency converter. Figure 12(b) shows the physical diagram of the hoist testing setup. Figure 12(c) shows the layout of measuring points, and a three-way accelerometer which can measure lowfrequency vibration (the frequency response range of $0-30 \mathrm{kHz}$ ) is fixed on the guide wheel bearing cover. The signal acquisition device (LMS SCADAS Mobile) is shown in Figure 12(d).
The hoist testing setup can obtain the vibration signal of the bearing under normal status, inner ring fault, outer ring fault, and rolling element fault. A single point was machined to simulate the failure, as shown in Figure 13. The bearing parameters are shown in Table 2.

In the test, the total lifting time is about $3.5 \mathrm{~s}$, and the vibration signal from $0.5 \mathrm{~s}$ to $3 \mathrm{~s}$ is considered to be generated during the constant speed lifting process. In this case, the rotating frequency $f_{r}$ was about $3.2 \mathrm{~Hz}$. The theoretical inner ring fault frequency $f_{i}$ is approximately $15.81 \mathrm{~Hz}$, outer ring fault frequency $f_{o}$ is $9.79 \mathrm{~Hz}$, and rolling element fault frequency $f_{b}$ is $6.43 \mathrm{~Hz}$. The sampling frequency $f_{s}$, sampling length $N$, estimation value of frequency difference $H$, and frequency bandwidth $2 \delta$ are set as $512 \mathrm{~Hz}, 1280$ samples, $0.3 \mathrm{~Hz}$, and $1 \mathrm{~Hz}$, respectively. The search range and the filter length range are in the interval [20 90] and [200 600], respectively.

\subsection{Automatic Identification of Different Fault Types}

3.3.1. Inner Ring Fault. The diagnosis process of bearing inner ring fault is shown in Figure 14. Figure 14(a) shows the inner ring fault vibration signal. The characteristic frequencies $(16,32,48 \mathrm{~Hz})$ representing the inner ring fault and the interference frequency $(53.20 \mathrm{~Hz})$ are obvious. Figure 14(b) shows the reconstructed signal at $c=0.03$. Compared with that of the raw signal, the amplitude of the characteristic frequency of $16 \mathrm{~Hz}$ increases obviously, and the interference frequency is wholly eliminated. Figure 14(c) shows the extracted characteristic frequencies, and all of them are near the theoretical inner fault frequency. Figure 14(d) shows the recognition result of the extracted feature frequency. It can be seen that $15.88 \mathrm{~Hz}$ obtained in first cycle corresponds to both the inner ring fault and the normal state and 15.79 and $15.76 \mathrm{~Hz}$ obtained in the second and third cycles only correspond to the inner ring fault, so as to determine the bearing inner ring failure.

3.3.2. Outer Ring Fault. Figure 15 shows the diagnosis process of the bearing outer ring fault. In Figure 15(a), the characteristic frequencies $(10,19.6,29.6 \mathrm{~Hz})$ of the original 


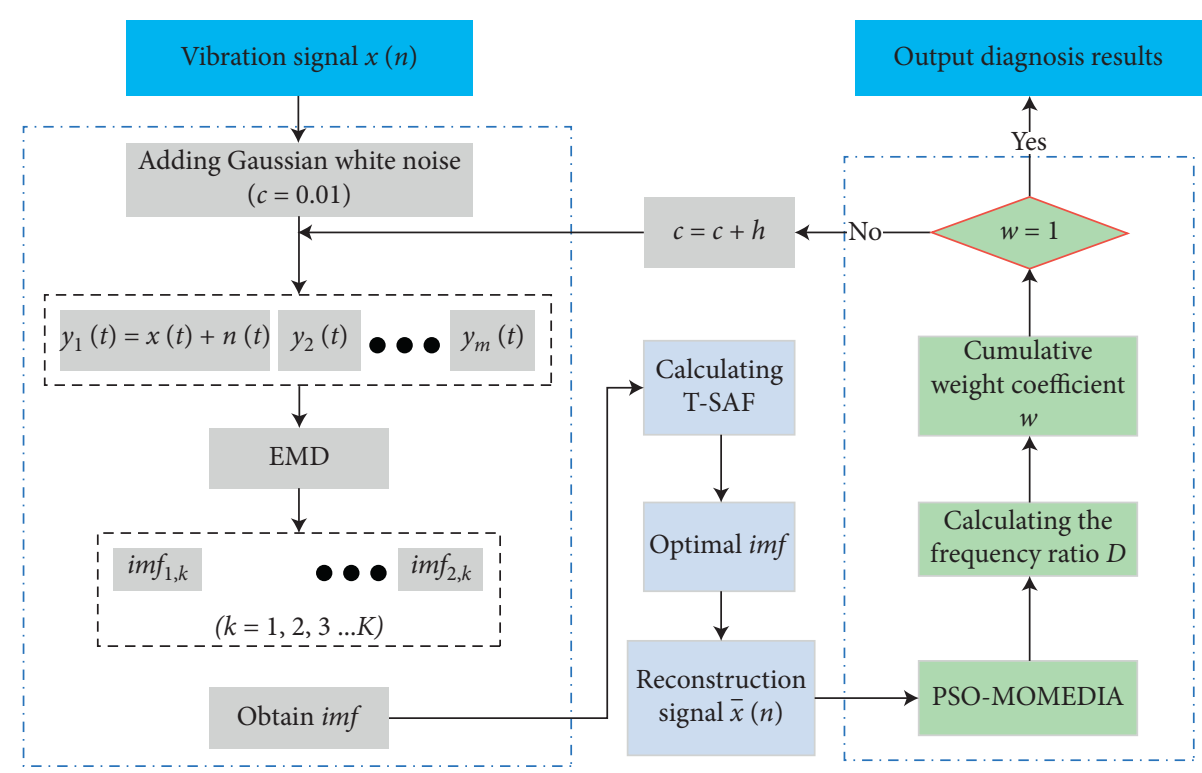

FIGURE 11: Steps of automatic diagnosis method for hoist bearing with unknown state.

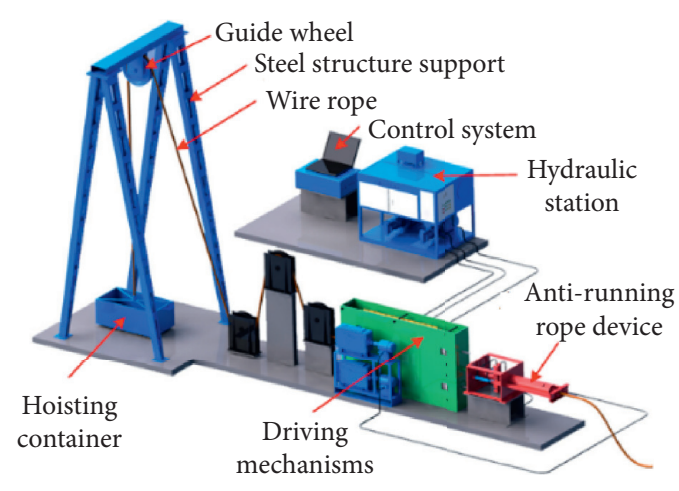

(a)

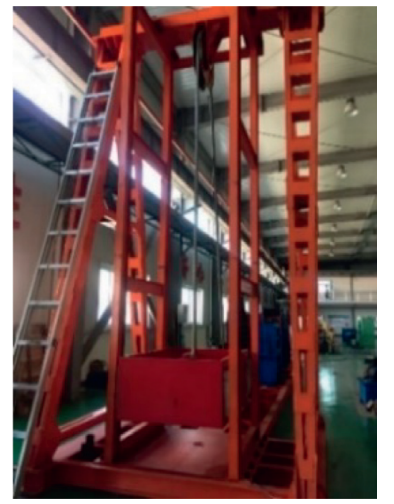

(b)

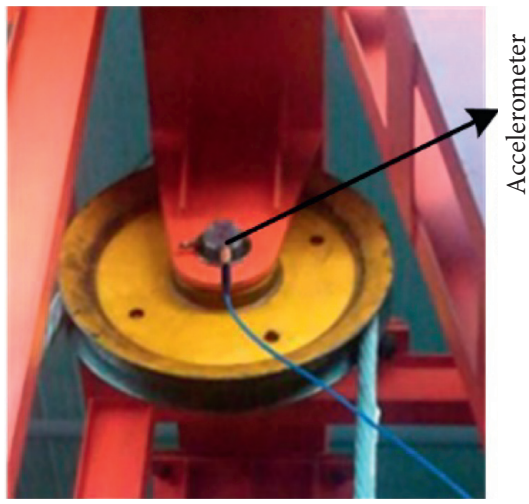

(c)

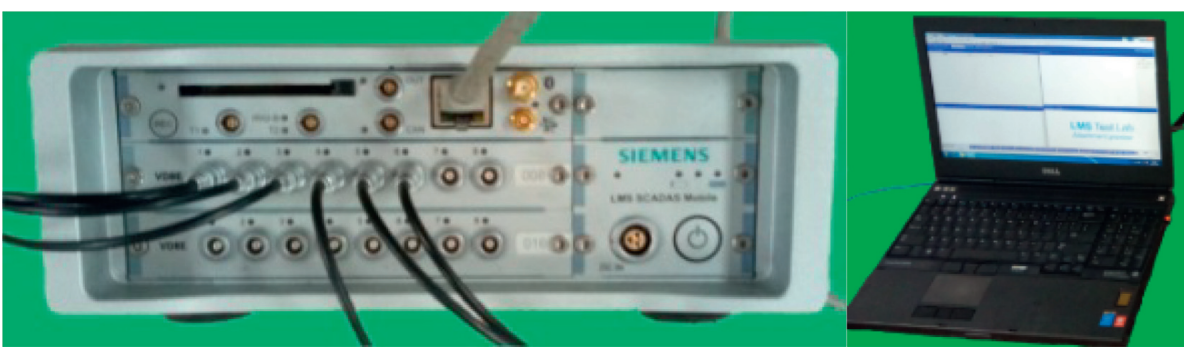

(d)

FIGURE 12: Hoist simulation testing setup: (a) equipment diagram; (b) physical map; (c) sensor's fixed position; (d) data acquisition equipment.

signal are close to the theoretical outer ring fault frequencies $\left(f_{o}=9.79 \mathrm{~Hz}, 2 f_{o}, 3 f_{o}\right)$, and the maximum amplitude $(10 \mathrm{~Hz})$ is $0.32 \mathrm{~m} / \mathrm{s}^{2}$. In the reconstructed signal, as shown in Figure 15(b), a large number of noise components are eliminated, and the amplitude of fault frequency $(10 \mathrm{~Hz})$ reaches $0.60 \mathrm{~m} / \mathrm{s}^{2}$. Figure 15 (c) shows that all the extracted characteristic frequencies are $9.81 \mathrm{~Hz}$. Figure 10 shows that the characteristic frequency only corresponds to the outer ring fault. So, the fault type is determined as the outer ring fault according to equation (5).

3.3.3. Rolling Element Fault. Figure 16 shows the processing result of the rolling element fault signal. Figures 16(a) and 16(b) show the original signal and reconstructed signal, respectively. The characteristic frequencies $(6.40,12.80 \mathrm{~Hz})$ 


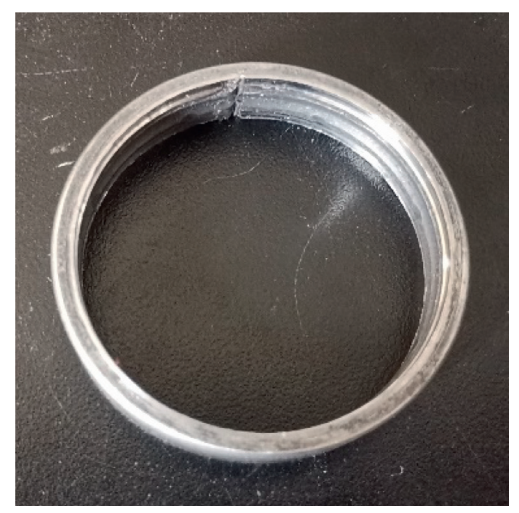

(a)

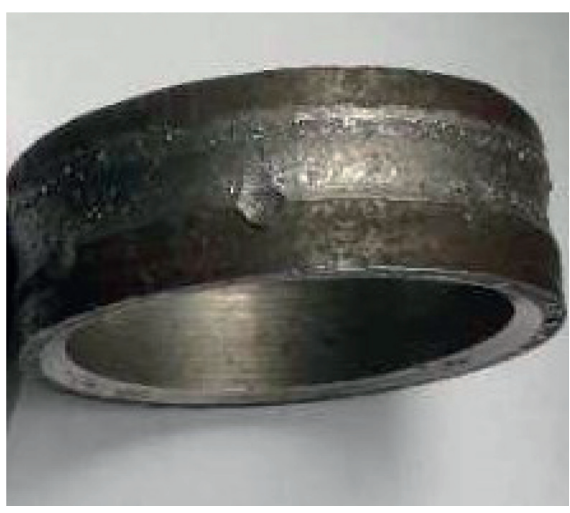

(b)

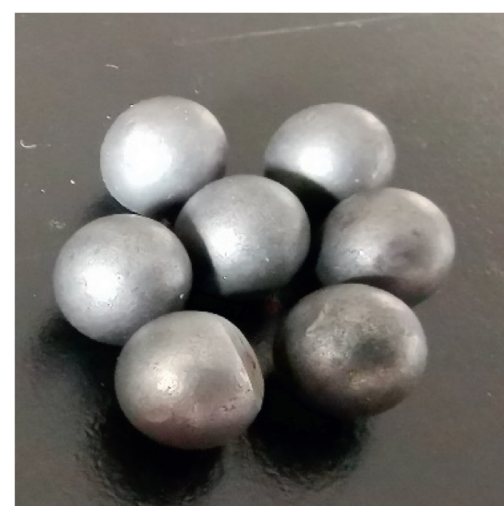

(c)

FIGURE 13: Different fault types of rolling bearing: (a) inner ring fault; (b) outer ring fault; (c) rolling element fault.

TABLE 2: The parameters of the rolling bearing.

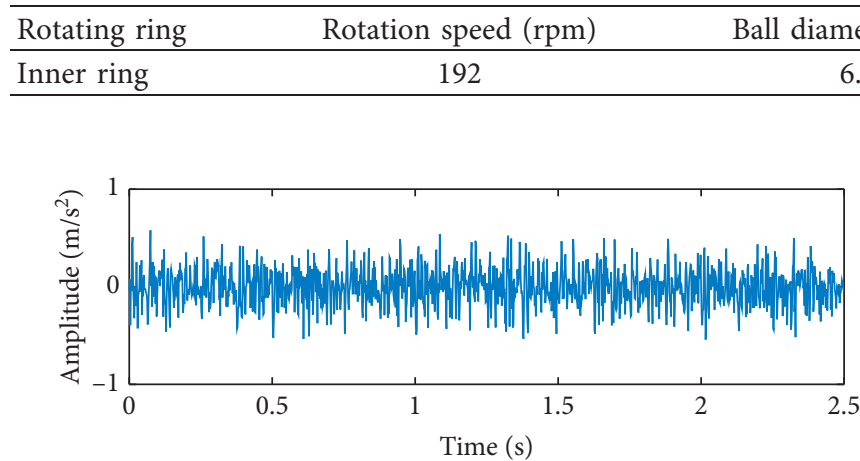

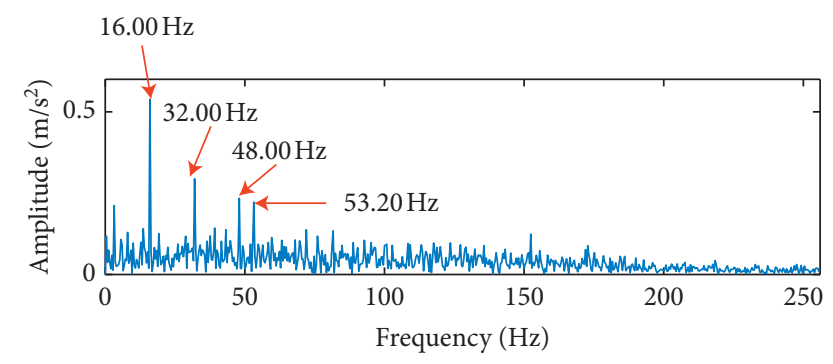

(a)

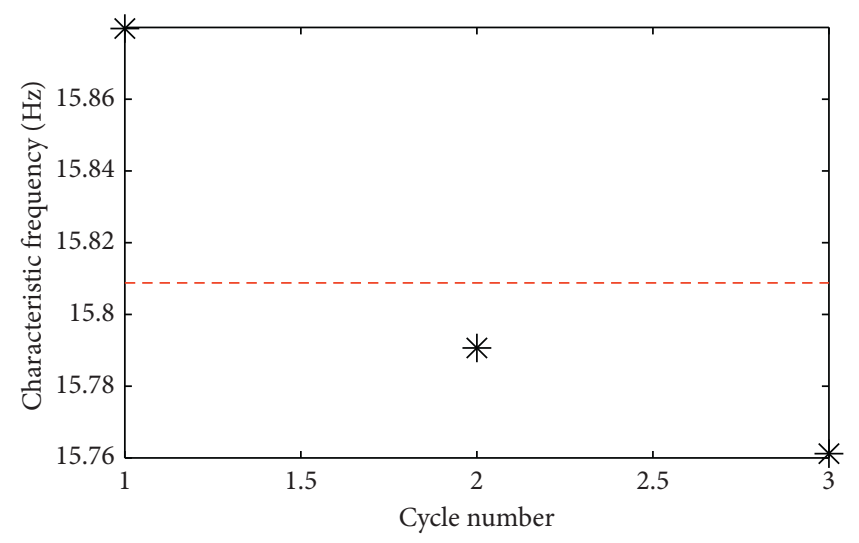

(c)
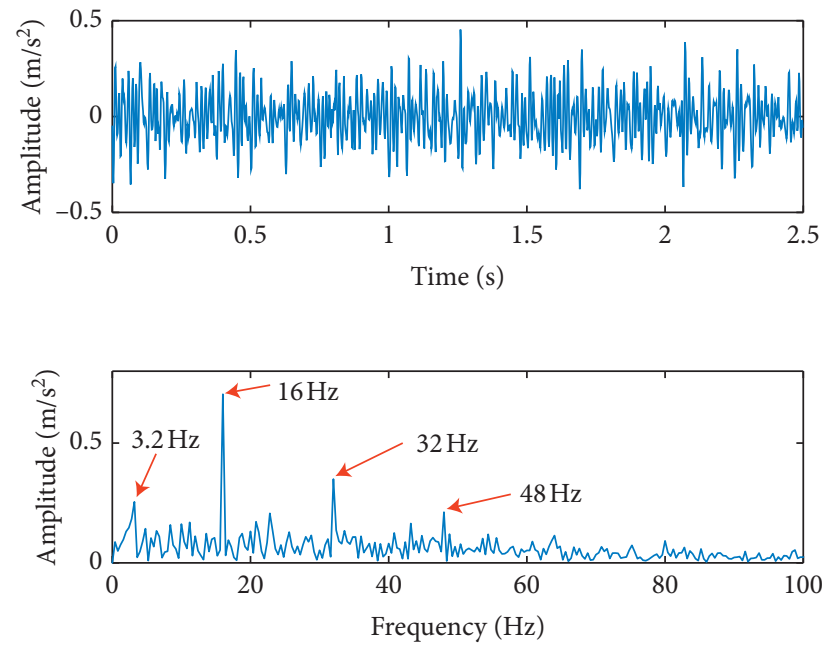

(b)

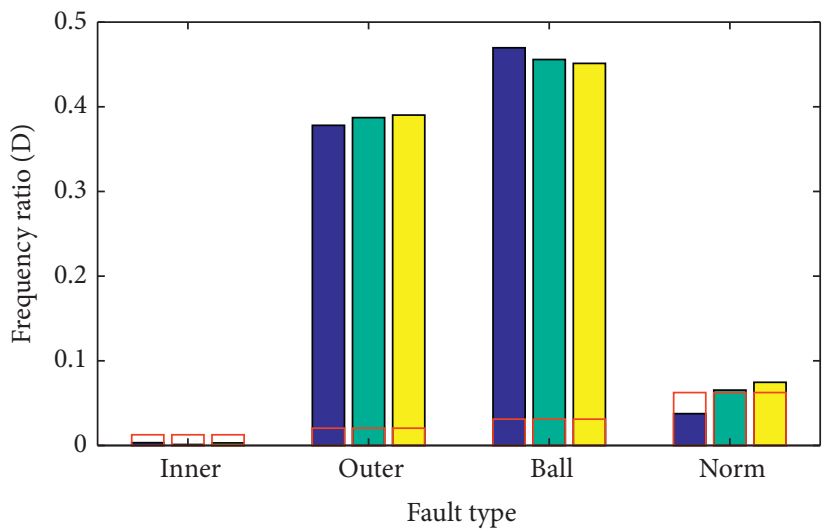

(d)

FIGURE 14: The diagnosis process of bearing inner ring fault: (a) the original signal; (b) the reconstructed signal; (c) characteristic frequency in each cycle; (d) the frequency ratio of fault types in each cycle. 

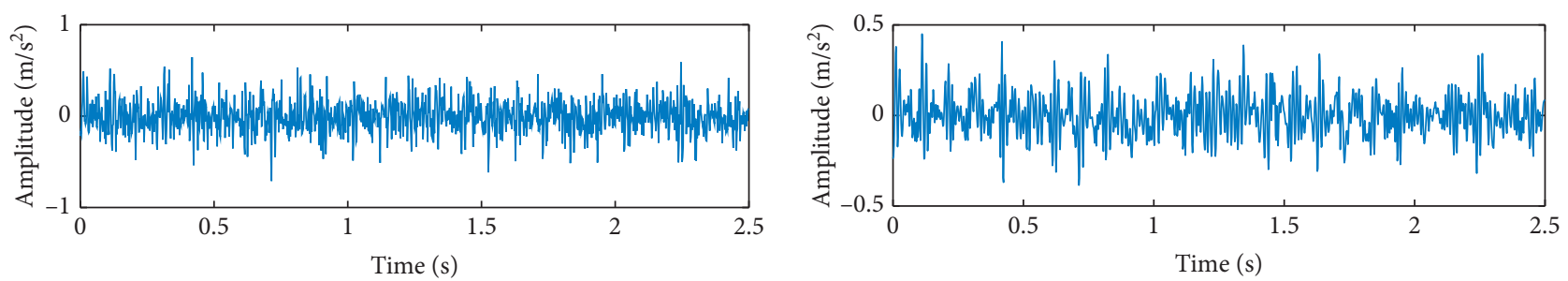

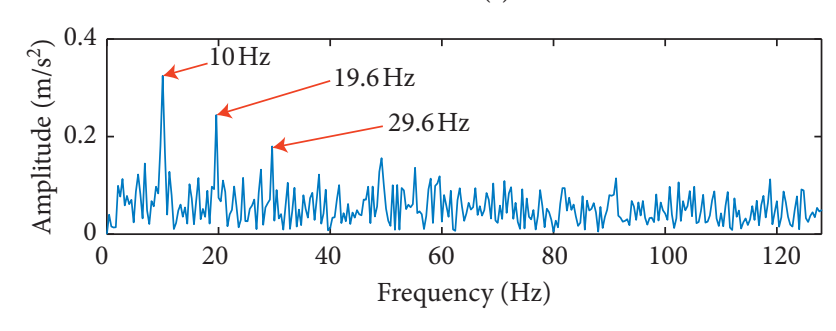

(a)

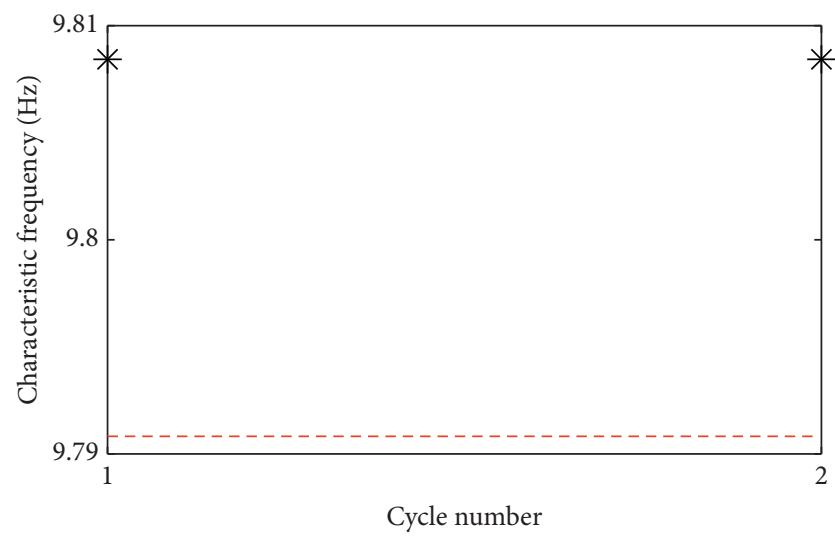

(c)

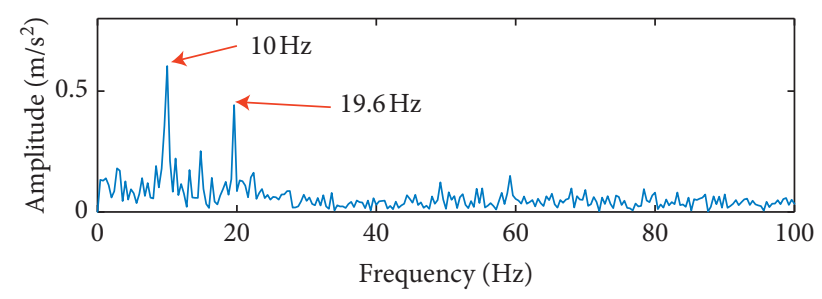

(b)

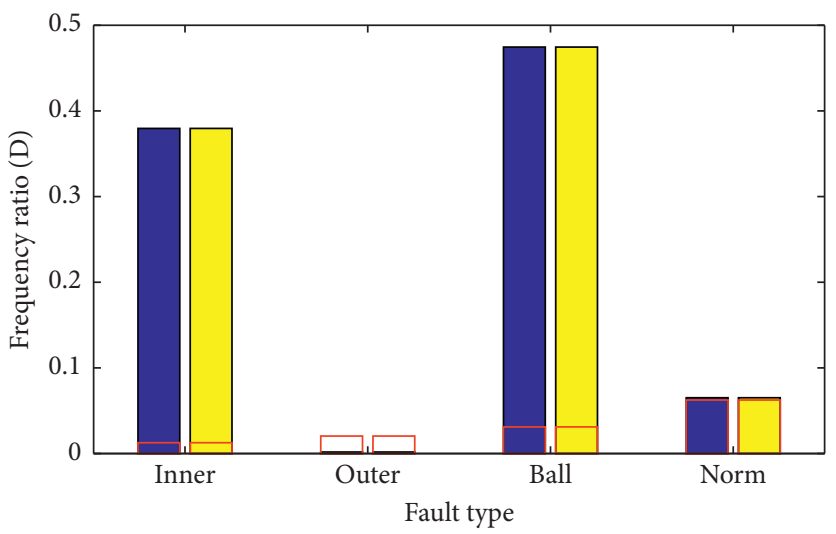

(d)

FIGURE 15: The diagnosis process of bearing outer ring fault: (a) the original signal; (b) the reconstructed signal; (c) characteristic frequency in each cycle; (d) the frequency ratio of fault types in each cycle.

in the reconstructed signal are more prominent, and the noise is significantly reduced. Figures $16(\mathrm{c})$ and $16(\mathrm{~d})$ show the extracted characteristic frequencies and the diagnosis results. In first, third, and sixth cycles, the frequency ratios meet the requirements of the rolling element fault; then, the fault type is diagnosed as a rolling element fault.

3.3.4. Normal State. Figure 17 shows the processing results of normal bearing vibration data. In Figure 17(a), the characteristic frequencies $(3.20,6.40 \mathrm{~Hz})$ make it difficult to judge whether the state is normal or the rolling element fault. In Figure 17(b), the rotation frequency and frequency doubling are more prominent. In Figures $17(\mathrm{c})$ and 17(d), when the characteristic frequency is close to $5 f_{r}=16 \mathrm{~Hz}$, it meets the bearing normal state threshold requirement, so the bearing state is considered normal.

3.3.5. Accuracy of This Method. The accuracy of the method is verified by processing 10 sets of experimental data for each bearing status, and the results are shown in Figure 18. In order to illustrate the detection effect of the proposed method, two indexes of recognition rate and accuracy rate are defined. The recognition rate is the accuracy of identifying normal or faulty bearings, and the accuracy rate is the correct proportion to identify the bearing state. For the inner ring fault, the recognition rate and accuracy rate are both $100 \%$. The recognition rate and accuracy rate for the outer ring fault are $100 \%$ and $90 \%$, respectively. The recognition rate and accuracy rate for the rolling element fault are $90 \%$ and $70 \%$, respectively. The recognition rate and accuracy rate for the bearing in the normal state are both $90 \%$.

Although this method fails to achieve a 100\% accuracy, misdiagnosis can be avoided by comparing the recognition results of multiple sets of vibration data. Besides, although this method takes a long time on average, the health of the hoist bearing is gradual in actual working conditions, so the real-time requirements are not strict. Based on the above analysis, this method cannot satisfy the requirements of field application.

\section{Field Test}

4.1. Field Environment and Equipment Installation. The algorithm is used to identify the state of head sheave bearing of the hoisting system under the actual working conditions. 

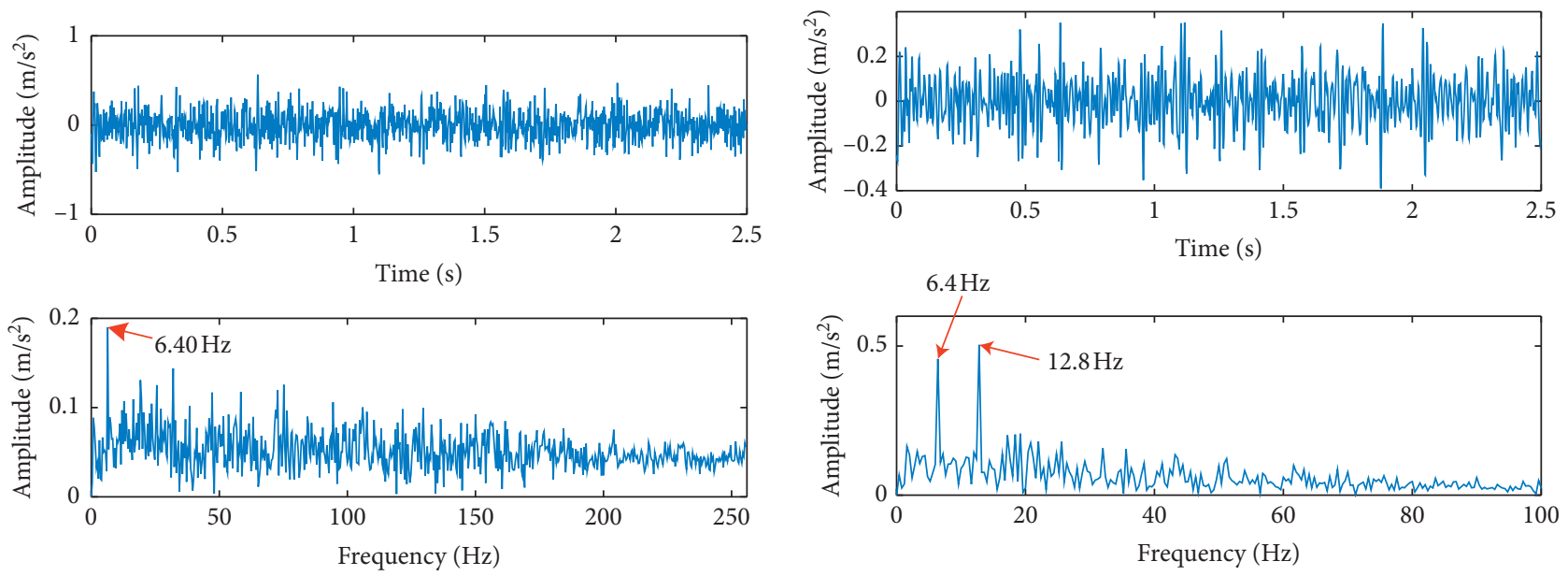

(a)

(b)

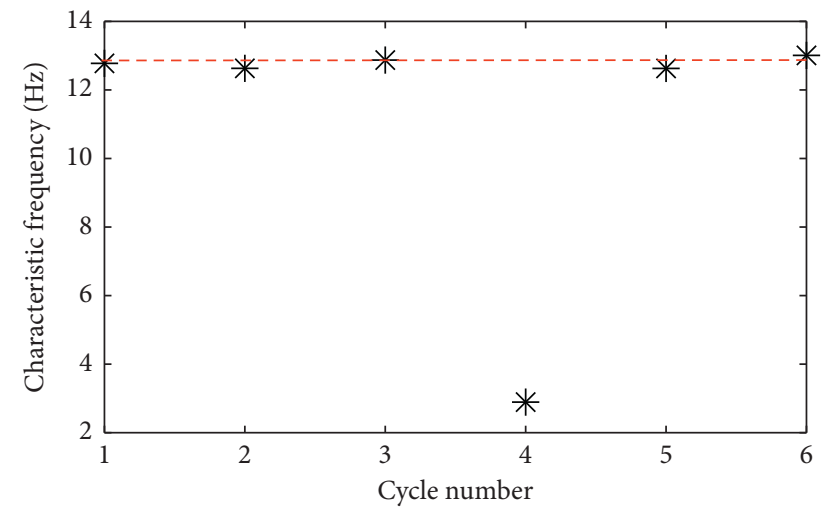

(c)

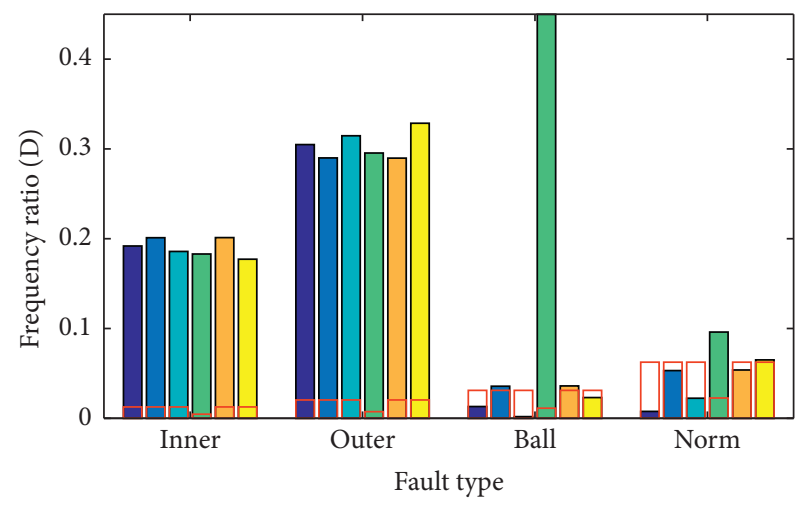

(d)

Figure 16: The diagnosis process of bearing rolling element fault: (a) the original signal; (b) the reconstructed signal; (c) characteristic frequency in each cycle; (d) the frequency ratio of fault types in each cycle.
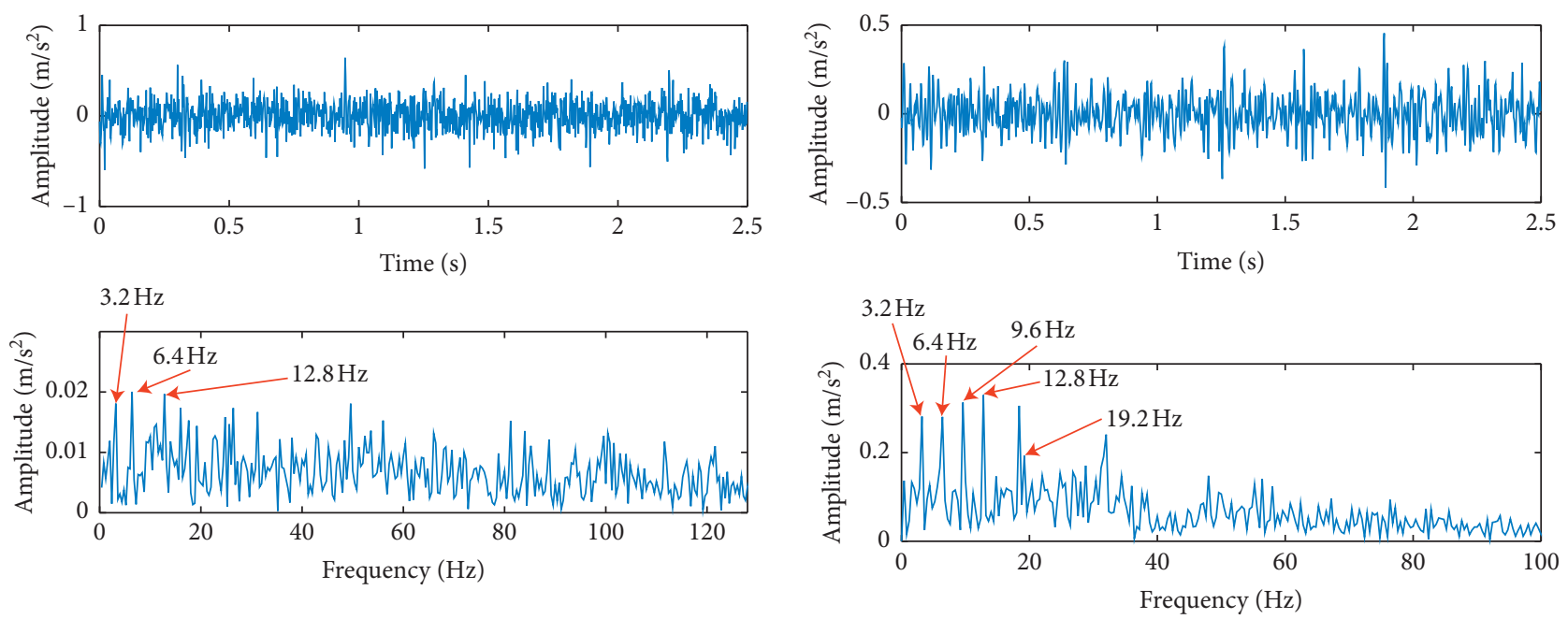

(a)

(b)

Figure 17: Continued. 


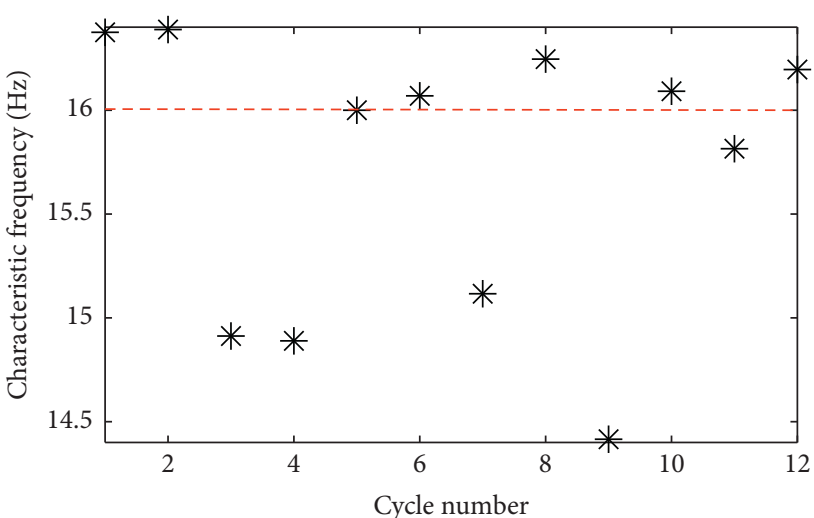

(c)

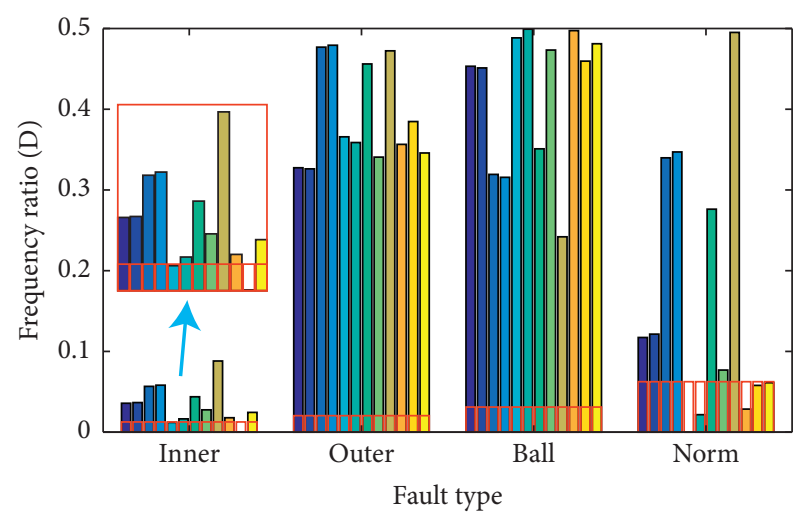

(d)

FIGURE 17: The diagnosis process of normal bearing: (a) the original signal; (b) the reconstructed signal; (c) characteristic frequency in each cycle; (d) the frequency ratio of fault types in each cycle.

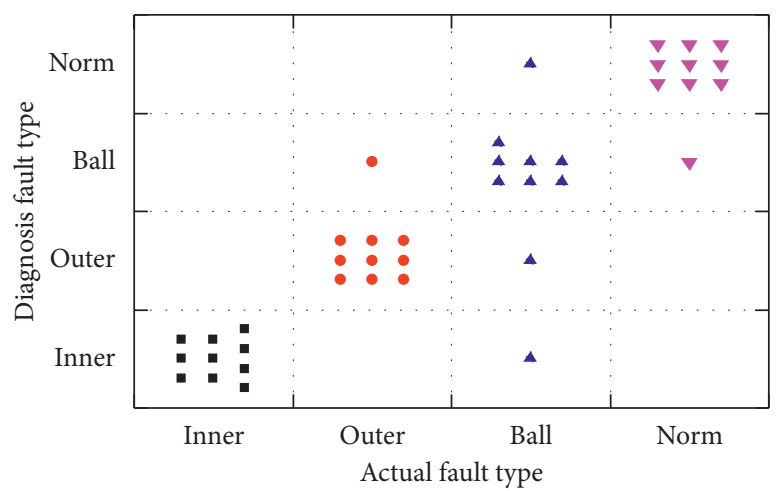

FigURE 18: Automatic diagnosis results of multiple signals.

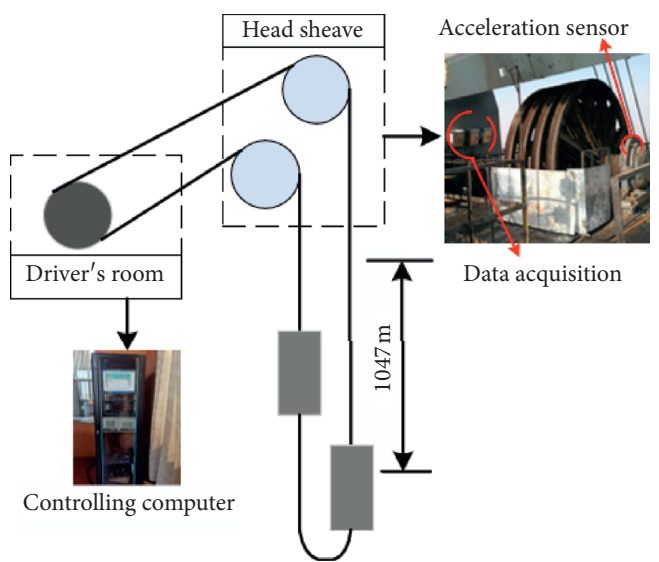

FIGURE 19: Installation diagram of the monitoring system.

The layout of the monitoring system is shown in Figure 19. The hoisting height is $1047 \mathrm{~m}$, the maximum hoist speed is $10.89 \mathrm{~m} / \mathrm{s}$, and the diameter of the head sheave is $4 \mathrm{~m}$. The bearings are inspected by workers to ensure that they are in good condition. The rotation frequency of the head sheave bearing is $0.87 \mathrm{~Hz}$, and the corresponding bearing fault frequency is consistent with that given in the simulation, $f_{i}=7.91 \mathrm{~Hz}, f_{o}=6.01 \mathrm{~Hz}$, and $f_{b}=3.13 \mathrm{~Hz}$. The sampling frequency $f_{s}$ is $256 \mathrm{~Hz}$, the estimate value $H$ is $0.2 \mathrm{~Hz}$, the frequency bandwidth $2 \delta$ is $1 \mathrm{~Hz}$, and the search range is in the interval [20 90]. The parameters of PSO are the same as experiment. In the field application, the vibration signal of the head sheave bearing is collected and wirelessly transmitted to the computer in the hoist driver cab.

4.2. Data Processing Results. The vibration signal is shown in Figure 20(a). The signal contains a lot of noise, and the peak 

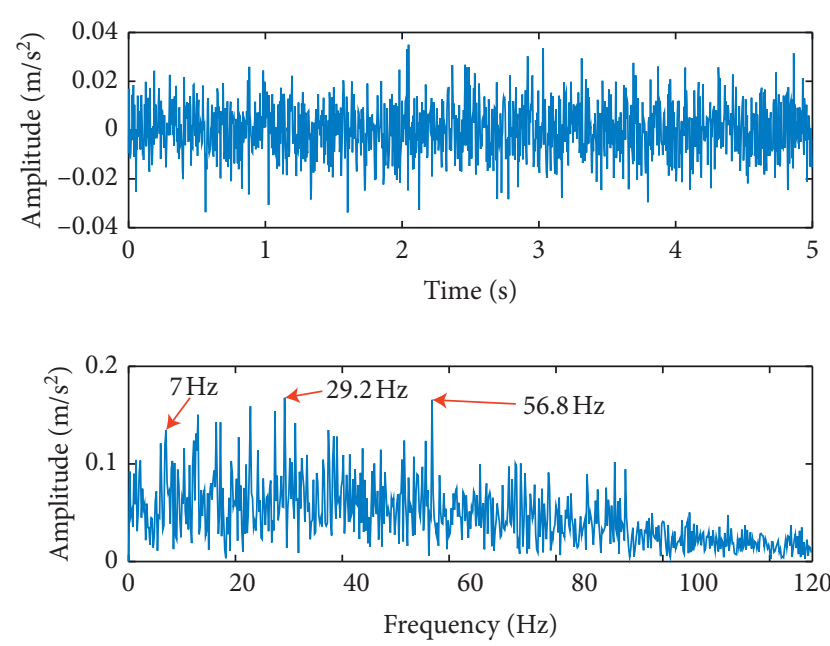

(a)

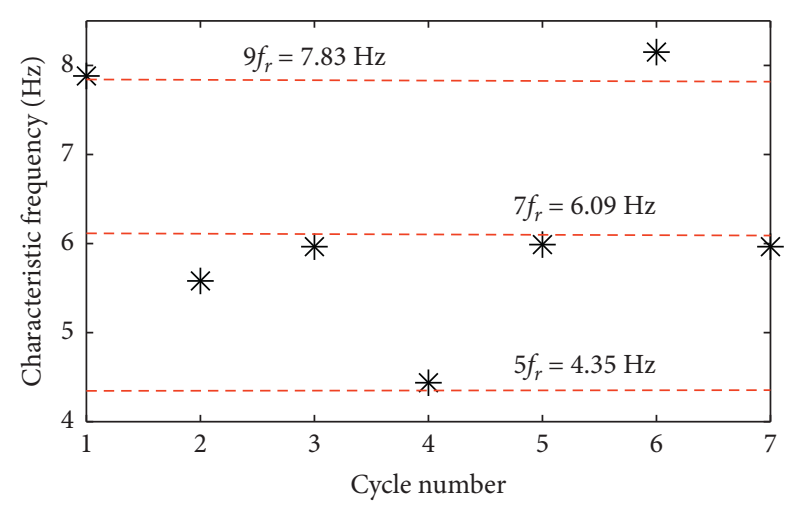

(c)
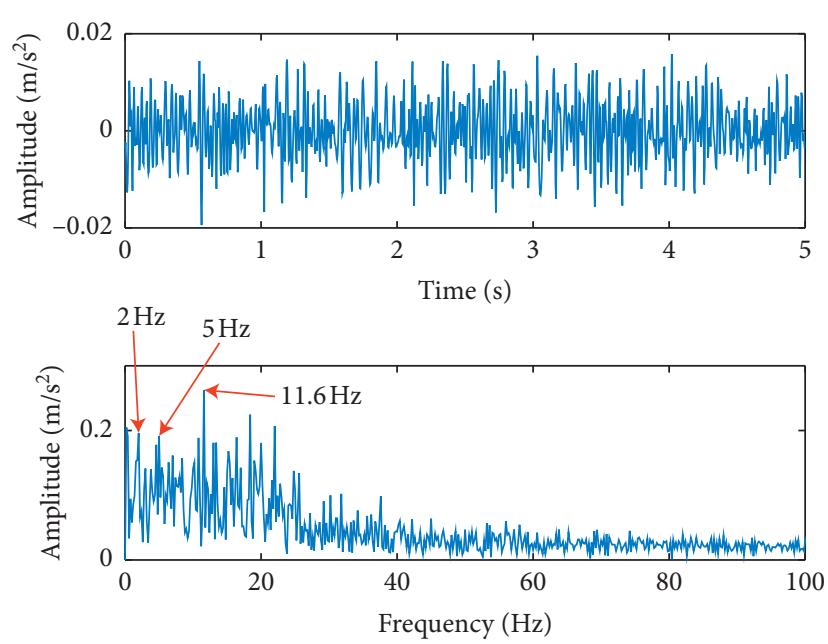

(b)

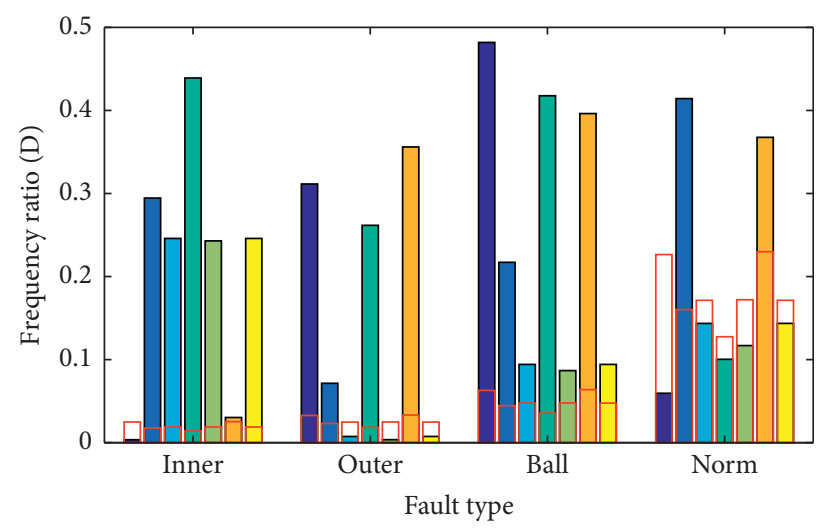

(d)

FIGURE 20: The diagnosis process of field vibration data: (a) the original signal; (b) the reconstructed signal; (c) characteristic frequency in each cycle; $(\mathrm{d})$ the frequency ratio of fault types in each cycle.

frequency is $29.2 \mathrm{~Hz}$. The reconstructed signal obtained when $c=0.07$ is given in Figure 20(b). The characteristic frequencies of $2.0\left(2 f_{r}=1.74\right), 5.0\left(6 f_{r}=5.22\right)$, and $11.6\left(13 f_{r}=11.31\right) \mathrm{Hz}$ that represent normal state are obvious. In Figures 20(c) and 20 (d), the extracted characteristic frequencies are distributed between 5 times and 9 times the rotation frequency, and the first, third, fourth, fifth, and seventh frequencies are effective characteristic frequencies which all meet the threshold requirements of the normal state. Therefore, it is determined that the bearing is in normal condition, which is consistent with the results given by the workers.

\section{Conclusions}

In this work, an adaptive fault diagnosis method composed of fault information integration and extracted feature evaluation is proposed. The feasibility of the proposed method is proved by the simulated signal, experimental signals, and on-site signal. The main results are as follows:

(1) The optimal IMF components can be selected effectively based on the T-SAF index. The reconstructed signal obtained by the CMF method can effectively reduce noise without losing fault information.
(2) The noise reduction effect of the MOMEDA method is greatly influenced by the filter length. The PSO method taking the fractal dimension as the objective function is used to select the optimal filter length. The cyclic recognition method can eliminate the influence caused by a larger search range as much as possible through the established evaluation system.

(3) The experimental results illustrate that the proposed method has good robustness. The field application results show that the method can effectively diagnose the bearing state under actual working conditions.

The proposed method can realize automatic recognition of the hoist bearing state, and the fault recognition rate of the method meets the requirements of the field application. It provides a reference for the diagnosis of other low-frequency bearings. In the next step, the accuracy of this method will be further improved.

\section{Data Availability}

The data used to support the findings of this study are given in the supplementary information files. 


\section{Conflicts of Interest}

The authors declare that they have no conflicts of interest.

\section{Authors' Contributions}

Tengyu Li conceived and designed the experiments. Ziming Kou and Waled Yahya performed the experiments. Tengyu $\mathrm{Li}$ and Juan $\mathrm{Wu}$ wrote the paper. All authors have read and approved the final manuscript.

\section{Acknowledgments}

This study was supported by the National Key Research and Development Plan (no. 2016YFC0600908) and Shanxi Province Applied Basic Research Project (grant no. 201801D121177).

\section{Supplementary Materials}

Simulation.txt contains the simulation signal data, corresponding to Figure 3 in the paper. Field.txt contains the collected vibration data of the hoist head sheave bearing in the field, corresponding to Figure 20 in the paper. ExpIn.txt is the vibration signal of the bearing with inner ring failure used in Figure 14 in the paper. ExpOu.txt is the vibration signal of the bearing with outer ring failure used in Figure 15 in the paper. ExpBa.txt is the vibration signal of the bearing with rolling element failure used in Figure 16 in the paper. ExpNo.txt is the vibration signal of the bearing with norm status used in Figure 17 in the paper. (Supplementary Materials)

\section{References}

[1] C. Ma, J. N. Yao, X. M. Xiao et al., "Fault diagnosis of head sheaves based on vibration measurement and data mining method," Advances in Mechanical Engineering, vol. 12, no. 7, pp. 1-8, 2020.

[2] J. Gu, Y. Peng, H. Lu, S. Cao, and B. Cao, "Fault diagnosis of spindle device in hoist using variational mode decomposition and statistical features," Shock and Vibration, vol. 2020, Article ID 8849513, 14 pages, 2020.

[3] Y.S. Ma and X. M. Xiao, "Dynamic analyses of hoisting ropes in a multi-rope friction mine hoist and determination of proper hoisting parameters," Journal of Vibroengineering, vol. 18, no. 5, pp. 2801-2817, 2016.

[4] D. Sandoval, U. Leturiondo, F. Pozo et al., "Low-speed bearing fault diagnosis based on permutation and spectral entropy measures," Applied Sciences-Basel, vol. 10, no. 13, pp. 1-15, 2020.

[5] J. Li, J. Jiang, X. Fan et al., "A new method for weak fault feature extraction based on improved MED," Shock and Vibration, vol. 2018, Article ID 9432394, 11 pages, 2018.

[6] J. B. Gai, J. X. Shen, Y. F. Hu et al., "An integrated method based on hybrid grey wolf optimizer improved variational mode decomposition and deep neural network for fault diagnosis of rolling bearing," Measurement, vol. 162, pp. 1-12, 2020.

[7] J. B. Hou, Y. X. Wu, H. Gong et al., "A novel intelligent method for bearing fault diagnosis based on EEMD permutation entropy and GG clustering," Applied SciencesBasel, vol. 10, no. 1, pp. 1-14, 2020.

[8] C. Y. Liu and K. Gryllias, "A semi-supervised support vector data description-based fault detection method for rolling element bearings based on cyclic spectral analysis," $\mathrm{Me}$ chanical Systems and Signal Processing, vol. 140, pp. 1-24, 2020.

[9] J. An, P. Ai, and D. Liu, "Deep domain adaptation model for bearing fault diagnosis with domain alignment and discriminative feature learning," Shock and Vibration, vol. 2020, Article ID 4676701, 14 pages, 2020.

[10] K. Dragomiretskiy and D. Zosso, "Variational mode decomposition," IEEE Transactions on Signal Processing, vol. 62, no. 3, pp. 531-544, 2014.

[11] J. Zheng, H. Pan, S. Yang, and J. Cheng, "Adaptive parameterless empirical wavelet transform based time-frequency analysis method and its application to rotor rubbing fault diagnosis," Signal Processing, vol. 130, pp. 305-314, 2017.

[12] L. Wang, Z. Liu, Q. Miao, and X. Zhang, “Time-frequency analysis based on ensemble local mean decomposition and fast kurtogram for rotating machinery fault diagnosis," $\mathrm{Me}$ chanical Systems and Signal Processing, vol. 103, pp. 60-75, 2018.

[13] T. Han, D. Jiang, and N. Wang, "The fault feature extraction of rolling bearing based on EMD and difference spectrum of singular value," Shock and Vibration, vol. 2016, Article ID 5957179, 14 pages, 2016.

[14] M. Kedadouche, M. Thomas, and A. Tahan, "A comparative study between empirical wavelet transforms and empirical mode decomposition methods: application to bearing defect diagnosis," Mechanical Systems and Signal Processing, vol. 81, pp. 88-107, 2016.

[15] X. Zhang, Y. Liang, J. Zhou, and Y. Zang, "A novel bearing fault diagnosis model integrated permutation entropy, ensemble empirical mode decomposition and optimized SVM," Measurement, vol. 69, pp. 164-179, 2015.

[16] I. I. E. Amarouayache, M. N. Saadi, N. Guersi et al., "Bearing fault diagnostics using EEMD processing and convolutional neural network methods," International Journal of Advanced Manufacturing Technology, vol. 107, no. 9-10, pp. 4077-4095, 2020.

[17] Y. Amirat, M. E. H. Benbouzid, T. Wang, K. Bacha, and G. Feld, "EEMD-based notch filter for induction machine bearing faults detection," Applied Acoustics, vol. 133, pp. 202-209, 2018.

[18] D. Zhen, J. C. Guo, Y. D. Xu et al., "A novel fault detection method for rolling bearings based on non-stationary vibration signature analysis," Sensors, vol. 19, no. 18, pp. 1-16, 2019.

[19] J. Lv and J. Yu, "Average combination difference morphological filters for fault feature extraction of bearing," $\mathrm{Me}$ chanical Systems and Signal Processing, vol. 100, pp. 827-845, 2018.

[20] F. Yang, Z. M. Kou, J. Wu et al., "Application of mutual information-sample entropy based MED-ICEEMDAN denoising scheme for weak fault diagnosis of hoist bearing," Entropy, vol. 20, no. 9, pp. 1-13, 2018.

[21] D. Y. Wu, J. W. Wang, H. Wang et al., "An automatic bearing fault diagnosis method based on characteristics frequency ratio," Sensors, vol. 20, no. 5, pp. 1-12, 2020.

[22] J. Li and J. Zhang, "Adaptive multiscale noise control enhanced stochastic resonance method based on modified EEMD with its application in bearing fault diagnosis," Shock and Vibration, vol. 2016, Article ID 1485412, 13 pages, 2016. 
[23] J. H. Yang, D. W. Huang, D. J. Zhou et al., "Optimal IMF selection and unknown fault feature extraction for rolling bearings with different defect modes," Measurement, vol. 157, pp. 1-15, 2020.

[24] M. Zhang, Z. Jiang, and K. Feng, "Research on variational mode decomposition in rolling bearings fault diagnosis of the multistage centrifugal pump," Mechanical Systems and Signal Processing, vol. 93, pp. 460-493, 2017.

[25] G. L. McDonald and Q. Zhao, "Multipoint optimal minimum entropy deconvolution and convolution fix: application to vibration fault detection," Mechanical Systems and Signal Processing, vol. 82, pp. 461-477, 2017.

[26] Y. Cheng, B. Chen, and W. Zhang, "Adaptive multipoint optimal minimum entropy deconvolution adjusted and application to fault diagnosis of rolling element bearings," IEEE Sensors Journal, vol. 19, no. 24, pp. 12153-12164, 2019.

[27] Y. Cheng, Z. Wang, W. Zhang, and G. Huang, "Particle swarm optimization algorithm to solve the deconvolution problem for rolling element bearing fault diagnosis," Isa Transactions, vol. 90, pp. 244-267, 2019.

[28] Z. Wang, W. Du, J. Wang et al., "Research and application of improved adaptive MOMEDA fault diagnosis method," Measurement, vol. 140, pp. 63-75, 2019.

[29] Y. G. Lei and M. J. Zuo, "Fault diagnosis of rotating machinery using an improved HHT based on EEMD and sensitive IMFs," Measurement Science and Technology, vol. 20, no. 12, pp. 1-12, 2009.

[30] Z. Wang, Z. Han, F. Gu, J. X. Gu, and S. Ning, "A novel procedure for diagnosing multiple faults in rotating machinery," Isa Transactions, vol. 55, pp. 208-218, 2015.

[31] L. Li, Y. Cui, R. Chen, L. Chen, and L. Wang, "Research on rolling bearing fault feature extraction based on singular value decomposition considering the singular component accumulative effect and teager energy operator," Shock and Vibration, vol. 2019, Article ID 3742512, 14 pages, 2019.

[32] D. Han, N. Zhao, and P. Shi, "Gear fault feature extraction and diagnosis method under different load excitation based on EMD, PSO-SVM and fractal box dimension," Journal of Mechanical Science and Technology, vol. 33, no. 2, pp. 487494, 2019. 